Dieter H. Hartmann is in the Department of Physics and Astronomy, Clemson University, Clemson, South Carolina 29634-0978, USA. e-mail: hdieter@clemson.edu

1. Gehrels, N. et al. Astrophys. J. 611, 1005-1020 (2004).

2. Klebesadel, R. W., Strong, I. B. \& Olson, R. A. Astrophys. J. 182, L85-L88 (1973).

3. Tagliaferri, G. et al. Nature 436, 985-988 (2005)

4. Kouveliotou, C. et al. Astrophys. J. 413, L101-L104 (1993).

5. Meegan, C. et al. Nature 355, 143-145 (1992)
6. Costa, E. et al. Nature 387, 783-785 (1997).

7. Metzger, M. Nature $\mathbf{3 8 7}, 878-880$ (1997).

8. MacFadyen, A. I. \& Woosley, S. E. Astrophys. J. 524, 262-289 (1999).

9. Rees, M. \& Meszaros, P. Astrophys. J. 430, L93-L96 (1994)

10. Rees, M. \& Meszaros, P. Mon. Not. R. Astron. Soc. 258, 41p-43p (1992).

11. Piran, T. Rev. Mod. Phys. 76, 1143-1210 (2004)

12. Kobayashi, S. et al. Astrophys. J. Lett. (submitted); preprint at www.arxiv.org/astro-ph/0506157 (2005)

13. Bloom, J. S., Frail, D. \& Kulkarni, S. R. Astrophys. J. 594, 674-683 (2003)

14. Ghirlanda, G. et al. Astrophys. J. 613, L13-L16 (2004).

\title{
CARDIOLOGY
}

\section{Rips repaired}

Richard A. Steinhardt

\section{In Duchenne muscular dystrophy, muscle cells die as a result of suffering many tiny membrane ruptures. A compound that increases membrane resealing can protect heart muscle cells from these effects.}

Degenerative muscle diseases are usually the result of defects in the cytoskeleton, the cell's internal scaffolding. This structure normally shields muscle cells from mechanical stress, protecting their membranes from tearing. People with Duchenne muscular dystrophy lack the protein dystrophin, which is an essential link in the complex of proteins that connect the cytoskeleton in the cell to a matrix of extracellular proteins. These buttresses across the membrane protect it from mechanical stress. Consequently, Duchenne dystrophic cells tend to get more rips in their membranes than do normal muscle cells. In this issue, Yasuda et al. (page 1025) ${ }^{1}$ show that the addition of a chemical that aids membrane resealing can preserve dystrophic heart muscle cells from such damage, and might help to maintain heart function in vivo.

Small rips in muscle cell membranes are normal, because these cells are under continuous mechanical stress. But in dystrophic muscle cells the frequency of these microdisruptions is greatly increased because their cytoskeleton cannot shield the membrane as effectively. Both normal and Duchenne dystrophic muscle cells can repair these tiny ruptures quickly, but not before calcium ions have flooded into the cell. This causes an increased calciumdependent breakdown of proteins, which in turn results in a persistent calcium influx in the vicinity of the resealed wound. Over time there is a gradual loss of calcium homeostasis, further activation of protein degradation, and eventual cell death ${ }^{2}$. As the dystrophic cells suffer many more rips than normal cells, cell death occurs more quickly.

Studies of Duchenne dystrophic cells have tended to concentrate on skeletal muscle rather than heart muscle, which under certain circumstances can be physiologically quite different. However, at least $15 \%$ of Duchenne sufferers die from heart failure. Yasuda et al. ${ }^{1}$ have now analysed dystrophic heart tissue, and they confirm that individual dystrophic heart muscle cells also show more stretch-mediated damage to their membrane than do normal cells. Just as in skeletal muscle cells, this is followed by loss of normal calcium homeostasis and cell death.

The authors next examined the effect of a surfactant called poloxamer 188 (also known as Pluronic F68) on the dystrophic cells. This compound has been used in cell culture since the early 1960 s to minimize mechanical damage from aeration and stirring of cell suspensions $^{3}$. It is now clear that poloxamer 188 acts by helping to seal up tears in the cell membrane. Cell membranes are under considerable tension from their attachment to the cytoskeleton, and this tension is sufficient to prevent passive resealing of rips in the lipid bilayer. Instead, an active process called exocytosis adds membrane by fusion with small membrane-bounded vesicles from within the cell to lower the membrane tension and close the break $^{4,5}$. Poloxamer 188 greatly accelerates resealing, even in the absence of exocytosis ${ }^{6}-$ apparently by lowering the tension from cytoskeletal attachments at the membrane $e^{5,6}$. This compound can insert directly into lipid monolayers ${ }^{7}$, and a similar process probably underlies its capacity to lower the tension in the bilayer lipid membrane.

Yasuda et al. ${ }^{1}$ found that adding poloxamer 188 to dystrophic cardiac cells in culture mitigates any stress-induced injuries to the membrane. To test whether the effect is significant in vivo, they used a mouse model of muscular dystrophy, subjecting the mice to a chemicalstress protocol that would usually cause heart failure. Strikingly, injecting poloxamer 188 into the mice before the start of the stress protocol blocked the heart failure. So membrane fragility, membrane disruption and the resulting abnormal calcium homeostasis found in dystrophic cardiac muscle can be corrected by an agent that rapidly reseals cell membranes. The authors propose that these results will have therapeutic implications for the treatment of muscular dystrophies.

A note of caution should be raised, however. Yasuda et al. demonstrate clear beneficial effects of adding poloxamer 188 to dystrophic heart muscle in the concentration range $0.5-1.25 \mathrm{mg}$ per $\mathrm{ml}$. But it is already known that the highest, most effective concentration is not well tolerated by healthy human volunteers given the compound for more than 44 hours $^{8}$. The lowest dose, which is tolerated for up to 72 hours in humans, may well have serious consequences if taken continuously over a lifetime. Lifelong treatment would be necessary because poloxamer 188 is useful only if it is present before the break in the membrane occurs. Only then can it speed the resealing of cell membrane, bypassing the normal process ${ }^{6}$. Nevertheless, although poloxamer 188 is not ready as a therapy for lifelong conditions, the results of Yasuda and colleagues do imply that further research on membrane sealants could be very useful, especially if harmful side effects could be avoided, or if brief treatment periods were found to be of lasting benefit.

There is another application, however, which might be of immediate benefit to all. Major surgery carries with it a significant risk of oxygen deprivation that can lead to damage to the patient's heart or brain, and death. It has been known for some time that using an agent that artificially reseals the membranes of oxygen-deprived heart cells will prevent cell death for at least 24 hours after oxygen withdrawal ${ }^{9}$. That study ${ }^{9}$ used targeted liposomes (artificial lipid vesicles) to reseal the membranes, which is not practical as a medical procedure. But, given that damaged cells are very rapidly resealed in the presence of poloxamer 188 (ref. 6), and that this occurs in vivo ${ }^{1}$, perhaps poloxamer 188 should be administered during high-risk surgery, at effective doses that are tolerated for 24 hours. The savings in terms of lives and the use of critical hospital resources might well be significant.

Richard A. Steinhardt is in the Department

of Molecular and Cell Biology,

University of California, Berkeley,

California 94720-3200, USA.

e-mail: ricksteinhardt@berkeley.edu

1. Yasuda, S. et al. Nature 436, 1025-1029 (2005).

2. Alderton, J. M. \& Steinhardt, R. A. Trends Cardiovasc. Med. 10, 268-272 (2000).

3. Runyan, W. S. \& Geyer, R. P. Proc. Soc. Exp. Biol. Med. 112, 1027-1030 (1963).

4. McNeil, P. L. \& Kirchhausen, T. Nature Rev. Mol. Cell Biol. 6 499-505 (2005).

5. Togo, T., Krasieva, T. B. \& Steinhardt, R. A. Mol. Biol. Cell 11, 4339-4346 (2000).

6. Togo, T., Alderton, J. M. \& Steinhardt, R. A. J. Cell Sci. 112, 719-731 (1999).

7. Maskarinec, S. A., Hannig, J., Lee, R. C. \& Lee, K. Y. C. Biophys. J. 82, 1453-1459 (2002).

8. Jewell, R. C., Khor, S. P., Kisor, D. F., LaCroix, K. A. \& Wargin, W. A. J. Pharm. Sci. 86, 808-812 (1997).

9. Khaw, B. A., Torchilin, V. P., Vural, I. \& Narula, J. Nature Med. 1, 1195-1198 (1995) 